\title{
Inverse Identification of Inputs of a Random Utility based Model Using Optimal Control
}

\author{
Parikshit Dutta \\ Department of Civil and Environmental Engineering, Duke University, Durham, NC. \\ email: parikshit.dutta@duke.edu.
}

\begin{abstract}
The random utility based multiregional input-output (RUBMRIO) model is used by several integrated land use and transportation models (ILUTMs) to forecast commodity flows in a region and for spatial allocation of production activities. It makes use of multiregional input-output models which are based on random utility theory. In this work, we use optimal control techniques to find optimal final demand and transportation costs of a RUBMRIO model, that would lead to a desired level of commodity flow and production costs.

At first, the RUBMRIO model is formulated as a discrete time dynamical system. It is shown, using Lyapunov argument, that the fixed point of the dynamical system is input-to-state stable (ISS). Then, a discrete time optimal control problem is formulated with states as commodity flows and production costs, and the final demand and transportation costs as control inputs. An optimization problem is then solved to obtain control inputs that would lead to desired commodity flows and production costs.

Further, the proposed methodology is applied to a numerical example. It is shown that the optimal control based method can achieve user specified commodity flows and production costs up to an acceptable accuracy level.

Keywords: Integrated land use and transportation, random utility based multiregional input-output models, Lyapunov stability, optimal control.
\end{abstract}

\section{Introduction}

Owing to constantly increasing awareness about environmental protection, urban sustainability has become a key policy issue over the years (Nash, 
2009). It is well known fact that integrated land use and transportation models play a key role in the context of urban sustainability (Spiekermann and Wegener, 2004; Black et al., 2002). Integrated land use and transport models (ILUTMs) offer invaluable analysis tools for planners working on transportation and urban projects. Analyzing the behavior of ILUTMs is important for policy makers in order to create sustainable cities for future. Hence, study and analysis of ILUTMs are key towards designing better urban environments (Dutta et al., 2012).

A number of ILUTMs, for example MEPLAN and TRANUS, make use of multiregional input-output models combined with random utility theory for allocation of activities and land use (Hunt, 1997; De La Barra, 1989). Since the input-output model varies spatially, between different geographical regions, they are called random utility based input-output (RUBMRIO) models. Typically they combine traditional input-output model with multinomial logit model for calculation of commodity flows and production prices between different regions (Zhao and Kockelman, 2004; Juri and Kockelman, 2004). The RUBMRIO model is usually solved iteratively, by applying a set of algebraic equations (Hunt, 1997). The outputs of the RUBMRIO model are commodity flows and production costs. The inputs are the transportation costs between different regions and the final demand values. Traditional use of RUBMRIO model is to predict the steady state commodity flows and production prices for given values of transportation costs, final demand and other input parameters. Particularly Kockelman et al. have used the model extensively to forecast land use and transportation scenarios in Texas and United States (Du and Kockelman, 2012; Ruiz Juri and Kockelman, 2006). They have also suggested variants of the original RUBMRIO model, in order to accommodate various objectives (Huang and Kockelman, 2010; Zhao and Kockelman, 2004; Juri and Kockelman, 2004). However, a methodology for inverse solution of the RUBMRIO model, where inputs are designed based on desired output values is missing from the literature.

In this work, we have solved an inverse problem to determine the inputs such that the desired output levels are met. The RUBMRIO model is represented as a nonlinear discrete time dynamical system with the states as the outputs of the RUBMRIO model and the controls as the inputs. An optimization problem is then solved to find the inputs that minimize the $\mathcal{L}_{2}$ norm between calculated RUBMRIO outputs and the desired output values.

Discrete time dynamical systems have been used to represent several "real world" systems over the years, ranging from queuing theory (Kleinrock, 1975) 
to cellular automata (Chopard and Droz, 1998). Several other applications include formal languages, temporal logic, perturbation analysis and computer simulation (Ben-Naoum et al., 1995; Sandefur, 1990). A nonlinear discrete dynamical system is represented as a sequence of states given by $\mathbf{x}(k) \in \mathfrak{R}^{n}$, where $k=1,2, \ldots, N$, whose evolution equation is given by,

$$
\mathbf{x}(k+1)=f(\mathbf{x}(k), \mathbf{u}(k))
$$

where $\mathbf{u}(k) \in \mathfrak{R}^{m}$ is the sequence of inputs to the dynamical system and $f: \mathfrak{R}^{n} \times \mathfrak{R}^{m} \mapsto \mathfrak{R}^{n}$ is a vector of bounded nonlinear functionals. The above system is called input-to-state stable (ISS), if the sequence of states $\mathbf{x}(k)<\infty$ given the input sequence $\mathbf{u}(k)<\infty \forall k=1, \ldots, N$ (Sontag and Wang, 1995; Jiang and Wang, 2001). It ensures that every state trajectory of the discrete system in eqn. (1) will be bounded if the control inputs are bounded. In the current work, we have shown via Lyapunov argument (Robinson, 2013), that the parameters of the RUBMRIO model can be designed such that it is ISS. Further the control inputs are obtained by solving an optimal control problem.

Optimal control deals with the problem of finding a control law for a given dynamical system such that a certain optimality criterion is achieved. An optimal control problem involves solving an optimization problem, which involves optimizing a cost functional that is a function of state and control variables, subject to the states and controls satisfying the equations of the dynamical system (Bryson and Ho, 1975). Optimal control has various applications ranging from astrodynamics, where it is used to optimize the entry trajectory of a satellite entering a planet's atmosphere (Singh et al., 2009; Dutta and Bhattacharya, 2010) to quantum mechanics, where it is used to optimize the path of a quantum mechanical system to its final state (Peirce et al., 1988). As far as ILUTMs are concerned, researchers have used Bayesian melding, to find optimal parameters in UrbanSim, an ILUTM (Sevcíková et al., 2007). As for models based on RUBMRIO theory, optimization techniques like MLE has been used by researchers to estimate parameters of TRANUS (Dutta et al., 2014). However, these work assume that the model involved is static, while estimating parameters. Finding the optimal inputs while taking into account the dynamical nature of RUBMRIO model has largely been ignored.

Supply-driven models, for example the Ghosh model (Dietzenbacher, 1997) exist in literature, which solves for the inputs like demand for a given supply. 
Hence these models are based on inverse of the demand-driven philosophy, on which the RUBMRIO model is based. The inverse identification method using optimal control solves for the demand while satisfying the RUBMRIO equations which are demand-driven. To the author's knowledge such a model is missing from the literature.

This paper has two key contributions. First, it is shown that the RUBMRIO model can be represented as a nonlinear discrete time dynamical system and the fixed point of the resulting dynamical system is ISS. Secondly, an optimal control problem is formulated, to find the inputs of the RUBMRIO model that will optimize a given cost functional. Further, the proposed optimal control methodology is applied to solve for the inputs of a RUBMRIO model that minimizes the $\mathcal{L}_{2}$ norm of the difference between calculated and desired state values of the outputs.

The rest of the paper is organized as follows: in Section 2 the RUBMRIO model is introduced and further in Section 3, it is shown that the discrete time dynamical system is ISS. In Section 4 the optimal control problem is formulated for various cost functionals. In Section 5 the results of application of the optimal control methodology to test problems is discussed and shown. Finally, in Section 6 conclusions are stated highlighting the future work to be done.

\section{Random Utility Based Multiregional Input-Output Model}

Input-output theory was originally proposed by Leontief, focusing on a single region's industry interactions with business expenditure (Leontief, 1953). The multiregional input-output (MIO) model extends the original input-output theory to include several regions (Leontief, 1986). The RUBMRIO model has since been developed, combining the MIO model to random utility theory (De La Barra, 1989). As stated earlier, the RUBMRIO model combines traditional input-output model with multinomial logit model for allocation of activities and land use. The final output is obtained by recursively solving a set of nonlinear algebraic equations. An intense mathematical description of the model can be found in Ref. (Zhao and Kockelman, 2004).

Input-output models characterize the interaction between market elements, aggregated into economic sectors. The model calculates flow of commodities or services between sectors, giving rise to productions and demand. For a multiregional model, flows can occur between different regions, adding a spatial element. The different regions are called geographical zones. If the 
region of interest is a city, then examples of economic sectors can be students or industry workers and even employments. Examples of geographical zones may be a neighborhood near an university or near a chemical factory.

The inputs to a multiregional input-output model are seen as the final demand for a sector in a given geographical zone and associated transportation cost that for a sector to be transported between two zones. From these inputs the intermediate demands are calculated. To meet the intermediate demands, production of services and commodities is required. The production costs and the commodity flow for a sector in a zone is essentially seen as the output of the model. If the multiregional input-output structure is determined by a multinomial logit model, we have the random utility based multiregional input-output model.

\subsection{Economic Definition of the Variables}

Let us consider, $M \in \mathbb{N}$ economic sectors and $J \in \mathbb{N}$ geographical regions. Let $x_{i j}^{n}$ be the commodity flow of sector $n$ from region $i$ to region $j$ and $b_{j}^{n}$ be the price of producing sector $n$ in region $j$. Also let $Y_{j}^{n}$ be the final demand of sector $n$ in region $j$ and $d_{i j}^{n}$ be the price of transporting one unit of sector $n$ from region $i$ to region $j$. Hence $n=1, \ldots, M$ and $i, j=1, \ldots, J$. As we

will see later, $x_{i j}^{n}$ and $b_{j}^{n}$ are the states and $Y_{j}^{n}$ and $d_{i j}^{n}$ are control inputs of the discrete time dynamical system. developed later in the paper.

The other variables that are germane to the RUBMRIO model are the technical coefficients $a_{j}^{m n}$ and the multinomial logit dispersion parameter $\lambda^{n}$. $a_{j}^{m n}$ represents the amount of sector $m$ (in dollars) required to produce an dollar worth of sector $n$ in region $j$ and $\lambda^{n}$ is a dispersion parameter for sector $n$, which is used in the multinomial logit equation of the RUBMRIO model. Typically both $a_{j}^{m n}>0, \forall m, n=1, \ldots, M$ and $j=1, \ldots, J$, and $\lambda^{n}>0, \forall n=1, \ldots, M$. An important point to note is that $\sum_{m=1}^{M} a_{j}^{m n}<1$, as the total amount of inputs required to produce a dollar of sector $n$ should be less than a dollar.

\subsection{Governing Equations}

The RUBMRIO model consists of several equations and variables having different economic interpretations. However, for simplicity, the development has been kept discreet and in this work, only the final equations of production cost and commodity flow have been presented. Detailed equations for the RUBMRIO model can be found in Appendix A. Interested readers are 
directed towards Ref. (Zhao and Kockelman, 2004) and Ref. (Hunt, 1997) for detailed description of the RUBMRIO model.

The evolution equation of the production price $b_{j}^{n}$ and the commodity flow $x_{i j}^{n}$ can be written as,

$$
\begin{aligned}
& b_{j}^{n}(k+1)=\sum_{m=1}^{M} a_{j}^{m n}\left[\sum_{i=1}^{J} \frac{\exp \left(-\lambda^{m}\left(b_{i}^{m}(k)+d_{i j}^{m}\right)\right)\left(b_{i}^{m}(k)+d_{i j}^{m}\right)}{\sum_{r=1}^{J} \exp \left(-\lambda^{m}\left(b_{r}^{m}(k)+d_{r j}^{m}\right)\right)}\right] \\
& x_{i j}^{n}(k+1)=\left[\sum_{m=1}^{M} a_{j}^{n m}\left(\sum_{l=1}^{J} x_{j l}^{m}(k)\right)+Y_{j}^{n}\right]\left[\frac{\exp \left(-\lambda^{n}\left(b_{i}^{n}(k)+d_{i j}^{n}\right)\right)}{\sum_{r=1}^{J} \exp \left(-\lambda^{n}\left(b_{r}^{n}(k)+d_{r j}^{n}\right)\right)}\right]
\end{aligned}
$$

The above system of equations (eqn. (2)) can be seen as a discrete dynamical system with states $x_{i j}^{n}$ and $b_{j}^{n}$, and $d_{i j}^{n}$ and $Y_{j}^{n}$ as the control inputs. $a_{j}^{m n}$ is assumed to be known and is constant. $\lambda^{n}$ is a parameter which is set by the user. It has been proven that the set of equation in eqn. (2) converge to a fixed point under certain regularity conditions on the states and the inputs (Zhao and Kockelman, 2004). As will be seen later, $\lambda^{n}$ is key in ensuring that the fixed point solution of the system is ISS.

\section{Input-to-State Stability of the Solution}

This section investigates the input-to-state stability (ISS) of the RUBMRIO dynamical system (Sontag and Wang, 1995). If a dynamical system is ISS, then it is ensured that for bounded control input the state trajectories of that dynamical system will be bounded. But first some basic definitions are presented which will help us show that the RUBMRIO model is ISS.

\subsection{Basic Definitions}

Let us consider the following definitions,

Definition 1 (Class $\mathcal{K}$ function). A continuous function $\gamma: \mathfrak{R}^{+} \mapsto \mathfrak{R}^{+}$is said to belong to class $\mathcal{K}$ if it is strictly increasing and $\gamma(0)=0$. 
Definition 2 (Class $\mathcal{K}_{\infty}$ function). A continuous function $\gamma: \mathfrak{R}^{+} \mapsto \mathfrak{R}^{+}$ is said to belong to class $\mathcal{K}_{\infty}$ if it is a class $\mathcal{K}$ function and $\lim _{s \rightarrow \infty} \gamma(s)=\infty$.

Definition 3 (ISS-Lyapunov function). (Sontag, 2008) A continuous function $V: \mathfrak{R}^{n} \mapsto \mathfrak{R}$ is called an ISS-Lyapunov function if the following holds.

1. There exists class $\mathcal{K}_{\infty}$ functions $\alpha_{1}, \alpha_{2}$, such that,

$$
\alpha_{1}(|s|) \leq V(|s|) \leq \alpha_{2}(|s|), \quad \forall s \in \mathfrak{R}^{n} .
$$

2. There exists class $\mathcal{K}_{\infty}$ function $\alpha_{3}$, and a class $\mathcal{K}$ function $\beta$ such that

$$
V(f(s, w))-V(s) \leq-\alpha_{3}(|s|)+\beta(|w|), \quad \forall s \in \mathfrak{R}^{n}, w \in \mathfrak{R}^{m}
$$

where $f(\cdot, \cdot)$ is the function in eqn. (1).

Lemma 1. (Jiang and Wang, 2001). If the dynamical system in eqn. (1) admits a continuous ISS-Lyapunov function then the fixed point of the system is ISS.

Theorem 1 (Mean value theorem for vector-valued functions.). (Hall and Newell, 1979). Given two vectors spaces $V_{1}$ and $V_{2}$ and a continuous map $\mathbf{h}$, we have

$$
\left|\mathbf{h}(\mathbf{v})-\mathbf{h}\left(\mathbf{v}^{\prime}\right)\right|<\sup _{\delta}\|\nabla \mathbf{h}(\delta)\| \cdot\left|\left(\mathbf{v}-\mathbf{v}^{\prime}\right)\right|, \quad|\mathbf{v}|<|\delta|<\left|\mathbf{v}^{\prime}\right|
$$

where $\mathbf{h}: V_{1} \mapsto V_{2}$ is a vector valued function, mapping vector space $V_{1}$ to space $V_{2}$, and $\delta, \mathbf{v}, \mathbf{v}^{\prime} \in V_{1} . \mathbf{h}$ is continuous in $\left[\mathbf{v}, \mathbf{v}^{\prime}\right]$ and differentiable in $\left(\mathbf{v}, \mathbf{v}^{\prime}\right) .|\cdot|$ is the norm defined in $V_{1}$ and $\|\cdot\|$ is the usual norm, defined for linear maps from $V_{1}$ to $V_{2}$.

Remark: Let the dimensions of $V_{1}$ and $V_{2}$ be $n_{v_{1}}$ and $n_{v_{2}}$ respectively. Then, we can write, $\nabla \mathbf{h}(\delta)=\{\nabla \mathbf{h}(\delta)\}_{n_{v_{2}} \times n_{v_{1}}}$. Let $\nabla \mathbf{h}(\delta)=\nabla h_{i, j}(\delta)$, where $i \in$ $\mathbb{N}\left[1, n_{v_{2}}\right]$ and $j \in \mathbb{N}\left[1, n_{v_{1}}\right]$. If $\mathbf{h}(\delta)=\left[h_{1}(\delta), h_{2}(\delta), \ldots, h_{n_{v_{2}}}(\delta)\right]^{\top}$ and $\delta=$ $\left[\delta_{1}, \delta_{2}, \ldots, \delta_{n_{v_{1}}}\right]^{\top}$, then $\nabla h_{i, j}(\delta)=\frac{\partial h_{i}}{\partial \delta_{j}}$. 


\subsection{ISS-Lyapunov Stability of the RUBMRIO Model}

We will use Lemma 1 to show that the discrete time system in eqn. (2) is an ISS system. We first consider a candidate Lyapunov function and then show that it is an ISS-Lyapunov function for the system in eqn. (2). Let the candidate Lyapunov function be given by,

$$
V\left(\left|b_{j}^{n}\right|,\left|x_{i j}^{n}\right|\right)=\sum_{j=1}^{J} \sum_{n=1}^{M}\left(b_{j}^{n}\right)^{2}+\sum_{i=1}^{J} \sum_{j=1}^{J} \sum_{n=1}^{M}\left(x_{i j}^{n}\right)^{2}
$$

Lemma 2. There exists $\mathcal{K}_{\infty}$ functions $\alpha_{1}$ and $\alpha_{2}$ such that $V(\cdot, \cdot)$ in eqn. (4) satisfies Property 1 of an ISS-Lyapunov function.

Proof: Let us consider two class $\mathcal{K}_{\infty}$ functions, $\alpha_{1}\left(\left|b_{j}^{n}\right|,\left|x_{i j}^{n}\right|\right)=\mathcal{C}_{1} \cdot V\left(\left|b_{j}^{n}\right|,\left|x_{i j}^{n}\right|\right)$ and $\alpha_{2}\left(\left|b_{j}^{n}\right|,\left|x_{i j}^{n}\right|\right)=\mathcal{C}_{2} \cdot V\left(\left|b_{j}^{n}\right|,\left|x_{i j}^{n}\right|\right)$. where $0<\mathcal{C}_{1}<1$ and $\mathcal{C}_{2}>1$ are two constants. Clearly,

$$
\alpha_{1}\left(\left|b_{j}^{n}\right|,\left|x_{i j}^{n}\right|\right) \leq V\left(\left|b_{j}^{n}\right|,\left|x_{i j}^{n}\right|\right) \leq \alpha_{2}\left(\left|b_{j}^{n}\right|,\left|x_{i j}^{n}\right|\right),
$$

which shows the Property 1 of ISS-Lyapunov function holds for $V(\cdot, \cdot)$ in eqn. (4).

In the context of RUBMRIO model, let us represent eqn. (1) as,

$$
\begin{aligned}
& \mathbf{b}(k+1)=\mathbf{g}_{1}(\mathbf{b}(k), \mathbf{x}(k) ; \mathbf{d}, \mathbf{Y} ; \Lambda) \\
& \mathbf{x}(k+1)=\mathbf{g}_{2}(\mathbf{b}(k), \mathbf{x}(k) ; \mathbf{d}, \mathbf{Y} ; \Lambda),
\end{aligned}
$$

where $\mathbf{b}(k), \mathbf{x}(k), \mathbf{d}, \mathbf{Y}$ and $\Lambda$ are vectorized forms of $b_{j}^{n}(k), x_{i j}^{n}(k), d_{i j}^{n}, Y_{j}^{n}$ and $\lambda^{n}$.

We will now show that Property 2 for an ISS-Lyapunov function holds for the candidate Lyapunov function. For notational simplicity, henceforth we will drop the discrete time index $k$.

Lemma 3. There exists $\Lambda$ such that the sequences of states generated by eqn. (5a) and eqn. (5b) are contractions in $\mathcal{L}_{2}$.

Proof: We will use the mean value theorem for vector-valued function (Theorem 1) to prove the current lemma. If the origin is a fixed point of the system in eqn. (5), then it is sufficient to show that $\exists \delta \in(\mathbf{0}, \mathbf{v})$ such that 
eqn. (3) holds and $\sup _{\delta}\|\nabla \mathbf{h}(\delta)\|<1$, where $\mathbf{v}$ is an arbitrary vector of states and controls.

Let $\mathbf{h}=\left[\mathbf{g}_{1}, \mathbf{g}_{2}\right]^{\top}$, also note that if $\mathbf{b}, \mathbf{x}, \mathbf{d}$ and $\mathbf{Y}$ are 0 then $\mathbf{h}$ is $\mathbf{0}$. Let, $\mathbf{v}^{\prime}=\mathbf{0}$ and $\mathbf{v}=[\mathbf{b}, \mathbf{x}, \mathbf{d}, \mathbf{Y}]^{\top}$, eqn. (3) can be written as,

$\left|\left[\begin{array}{l}\mathbf{g}_{1}(\mathbf{b}, \mathbf{x} ; \mathbf{d}, \mathbf{Y}) \\ \mathbf{g}_{2}(\mathbf{b}, \mathbf{x} ; \mathbf{d}, \mathbf{Y})\end{array}\right]\right|<\sup _{\delta}\left\|\left[\begin{array}{cccc}\frac{\partial \mathbf{g}_{1}}{\partial \mathbf{b}} & \frac{\partial \mathbf{g}_{1}}{\partial \mathbf{x}} & \frac{\partial \mathbf{g}_{1}}{\partial \mathbf{d}} & \frac{\partial \mathbf{g}_{1}}{\partial \mathbf{Y}} \\ \frac{\partial \mathbf{g}_{2}}{\partial \mathbf{b}} & \frac{\partial \mathbf{g}_{2}}{\partial \mathbf{x}} & \frac{\partial \mathbf{g}_{2}}{\partial \mathbf{d}} & \frac{\partial \mathbf{g}_{2}}{\partial \mathbf{Y}}\end{array}\right]_{\delta}\right\||\cdot|\left[\begin{array}{llll}\mathbf{b} & \mathbf{x} & \mathbf{d} & \mathbf{Y}\end{array}\right]^{\top} \mid$,

where $\mathbf{v}^{\prime}<|\delta|<\mathbf{v}$.

The partial derivatives in eqn. (6), can be calculated from eqn. (2). Assuming,

$$
P_{i j}^{n}=\frac{\exp \left(-\lambda^{n}\left(b_{i}^{n}+d_{i j}^{n}\right)\right)}{\sum_{l=1}^{J} \exp \left(-\lambda^{n}\left(b_{l}^{n}+d_{l j}^{n}\right)\right)},
$$

it is easy to see that (Zhao and Kockelman, 2004),

$$
\begin{aligned}
\frac{\partial \mathbf{g}_{1}}{\partial \mathbf{b}}=\frac{\partial g_{1 j}^{n}}{\partial b_{j_{1}}^{n_{1}}} & =\frac{\partial}{\partial b_{j_{1}}^{n_{1}}}\left\{\sum_{m=1}^{M} a_{j}^{m n}\left(\sum_{i=1}^{J} \frac{\exp \left(-\lambda^{m}\left(b_{i}^{m}+d_{i j}^{m}\right)\right)\left(b_{i}^{m}+d_{i j}^{m}\right)}{\sum_{r=1}^{J} \exp \left(-\lambda^{m}\left(b_{r}^{m}+d_{r j}^{m}\right)\right)}\right)\right\} \\
& =a_{j}^{n_{1} n} P_{j_{1} j}^{n_{1}}\left[1-\lambda^{n_{1}}\left\{b_{j_{1}}^{n_{1}}+d_{j_{1} j}^{n_{1}}-\sum_{l=1}^{J} P_{l j}^{n_{1}}\left(b_{l}^{n_{1}}+d_{l j}^{n_{1}}\right)\right\}\right] .
\end{aligned}
$$

Similarly, we have,

$$
\begin{aligned}
\frac{\partial \mathbf{g}_{1}}{\partial \mathbf{x}} & =\frac{\partial g_{1 j}^{n}}{\partial x_{i_{1} j_{1}}^{n_{1}}}=0 \\
\frac{\partial \mathbf{g}_{1}}{\partial \mathbf{d}} & =\frac{\partial g_{1 j}^{n}}{\partial d_{i_{1} j_{1}}^{n_{1}}}=\frac{\partial}{\partial d_{i_{1} j_{1}}^{n_{1}}}\left\{\sum_{m=1}^{M} a_{j}^{m n}\left(\sum_{i=1}^{J} P_{i j}^{m}\left(b_{i}^{m}+d_{i j}^{m}\right)\right)\right\} \\
& = \begin{cases}a_{j_{1}}^{n_{1} n} P_{i_{1} j_{1}}^{n_{1}}\left[1-\lambda^{n_{1}}\left\{b_{i_{1}}^{n_{1}}+d_{i_{1} j_{1}}^{n_{1}}-\sum_{l=1}^{J} P_{l j_{1}}^{n_{1}}\left(b_{l}^{n_{1}}+d_{l j_{1}}^{n_{1}}\right)\right\}\right], & \text { if, } j=j_{1}(8 \mathrm{~b}) \\
0, & \text { otherwise. }\end{cases} \\
\frac{\partial \mathbf{g}_{1}}{\partial \mathbf{Y}} & =\frac{\partial g_{1 j}^{n}}{\partial Y_{j_{1}}^{n_{1}}}=0
\end{aligned}
$$


Also,

$$
\begin{aligned}
& \frac{\partial \mathbf{g}_{2}}{\partial \mathbf{b}}=\frac{\partial g_{2 i j}^{n}}{\partial b_{j_{1}}^{n_{1}}}= \begin{cases}\lambda^{n_{1}} P_{j_{1 j} j}^{n_{1}}\left(P_{j_{1} j}^{n_{1}}-1\right)\left[\sum_{m=1}^{M} a_{j}^{n_{1} m}\left(\sum_{l=1}^{J} x_{j l}^{m}\right)+Y_{j}^{n_{1}}\right], & \text { if, } i=j_{1}, n=n_{1}, \\
\lambda^{n_{1}} P_{i j}^{n_{1}} P_{j_{1} j}^{n_{1}}\left[\sum_{m=1}^{M} a_{j}^{n_{1} m}\left(\sum_{l=1}^{J} x_{j l}^{m}\right)+Y_{j}^{n_{1}}\right], & \text { if, } n=n_{1}, \\
0, & \text { otherwise. }\end{cases} \\
& \frac{\partial \mathbf{g}_{2}}{\partial \mathbf{d}}=\frac{\partial g_{2 i j}^{n}}{\partial d_{i_{1} j_{1}}^{n_{1}}}= \begin{cases}\lambda^{n_{1}} P_{i_{1} j_{1}}^{n_{1}}\left(P_{i_{1} j_{1}}^{n_{1}}-1\right)\left[\sum_{m=1}^{M} a_{j_{1}}^{n_{1} m}\left(\sum_{l=1}^{J} x_{j_{1} l}^{m}\right)+Y_{j_{1}}^{n_{1}}\right], & \text { if, } i=i_{1}, j=j_{1}, n=n_{1} \\
\lambda^{n_{1}} P_{i j_{1}}^{n_{1}} P_{i_{1} j_{1}}^{n_{1}}\left[\sum_{m=1}^{M} a_{j_{1}}^{n_{1} m}\left(\sum_{l=1}^{J} x_{j_{1} l}^{m}\right)+Y_{j_{1}}^{n_{1}}\right], & \text { if, } j=j_{1},, n=n_{1}, \\
0, & \text { otherwise. }\end{cases} \\
& \frac{\partial \mathbf{g}_{2}}{\partial \mathbf{x}}=\frac{\partial g_{2 i j}^{n}}{\partial x_{i_{1} j_{1}}^{n n_{1}}}= \begin{cases}a_{i_{1}}^{n n_{1}} P_{i i_{1}}^{n}, & \text { if, } j=i_{1}, \\
0, & \text { otherwise. }\end{cases} \\
& \frac{\partial \mathbf{g}_{2}}{\partial \mathbf{Y}}=\frac{\partial g_{2 i j}^{n}}{\partial Y_{j_{1}}^{n_{1}}}= \begin{cases}P_{i j_{1}}^{n_{1}}, & \text { if, } j=j_{1}, n=n_{1}, \\
0, & \text { otherwise. }\end{cases}
\end{aligned}
$$

As we need to show that state sequences in eqn. (5) are contractions in $\mathcal{L}_{2}$, we will assume that the vector spaces $V_{1}$ and $V_{2}$ are equipped with $\mathcal{L}_{2}$ norm. Hence the operator norm $\|\cdot\|$ for the Jacobian matrix in eqn. (6) is the $\mathcal{L}_{2}$ norm or the spectral norm. But from the equivalence of norms we know that for a matrix $A \in \mathfrak{R}^{m \times n}$, we have $\|A\|_{2}<\sqrt{m}\|A\|_{\infty}$, where subscripts represent appropriate norms. Hence eqn. (6) can be rewritten as,

$$
\left|\left[\begin{array}{l}
\mathbf{g}_{1}(\mathbf{b}, \mathbf{x} ; \mathbf{d}, \mathbf{Y}) \\
\mathbf{g}_{2}(\mathbf{b}, \mathbf{x} ; \mathbf{d}, \mathbf{Y})
\end{array}\right]\right|_{2}<\sqrt{4 M+6 J} \sup _{\delta} \underbrace{\left\|\left[\begin{array}{cccc}
\frac{\partial \mathbf{g}_{1}}{\partial \mathbf{b}} & \frac{\partial \mathbf{g}_{1}}{\partial \mathbf{x}} & \frac{\partial \mathbf{g}_{1}}{\partial \mathbf{d}} & \frac{\partial \mathbf{g}_{1}}{\partial \mathbf{Y}} \\
\frac{\partial \mathbf{g}_{2}}{\partial \mathbf{b}} & \frac{\partial \mathbf{g}_{2}}{\partial \mathbf{x}} & \frac{\partial \mathbf{g}_{2}}{\partial \mathbf{d}} & \frac{\partial \mathbf{g}_{2}}{\partial \mathbf{Y}}
\end{array}\right]_{\delta}\right\|_{\infty}}_{\|\nabla \mathbf{h}(\delta)\|_{\infty}} \cdot\left|\left[\begin{array}{c}
\mathbf{b} \\
\mathbf{x} \\
\mathbf{d} \\
\mathbf{Y}
\end{array}\right]\right|_{2}
$$

Considering the $\infty$-norm of the Jacobian matrix in eqn. (10), we have,

$$
\|\nabla \mathbf{h}(\delta)\|_{\infty}=\max _{1 \leq i \leq n_{v_{2}}} \sum_{j=1}^{n_{v_{1}}}\left|\nabla h_{i, j}(\delta)\right|=\max _{\substack{n \in \mathbb{N}[1, M] \\ i, j \in \mathbb{N}[1, J]}}\left({ }^{1} S_{j}^{n},{ }^{2} S_{i j}^{n}\right)
$$

where, $|\cdot|$ represents the absolute value. Let

$$
C_{j}^{m}(k+1)=\sum_{n=1}^{M} a_{j}^{m n} X_{j}^{n}(k+1)+Y_{j}^{m}
$$


then we have,

$$
\begin{aligned}
{ }^{1} S_{j}^{n}= & \sum_{n_{1}=1}^{M} \sum_{j_{1}=1}^{J}\left|a_{j}^{n_{1} n} P_{j_{1} j}^{n_{1}}\left[1-\lambda^{n_{1}}\left\{b_{j_{1}}^{n_{1}}+d_{j_{1} j}^{n_{1}}-\sum_{l=1}^{J} P_{l j}^{n_{1}}\left(b_{l}^{n_{1}}+d_{l j}^{n_{1}}\right)\right\}\right]\right| \\
& +\sum_{n_{1}=1}^{M} \sum_{i_{1}=1}^{J}\left|a_{j}^{n_{1} n} P_{i_{1} j}^{n_{1}}\left[1-\lambda^{n_{1}}\left\{b_{i_{1}}^{n_{1}}+d_{i_{1} j}^{n_{1}}-\sum_{l=1}^{J} P_{l j}^{n_{1}}\left(b_{l}^{n_{1}}+d_{l j}^{n_{1}}\right)\right\}\right]\right|, \\
{ }^{2} S_{i j}^{n}= & \left|\lambda^{n} P_{i j}^{n}\left(P_{i j}^{n}-1\right) C_{j}^{n}\right|+\sum_{\substack{j_{1}=1 \\
j_{1} \neq i}}^{M}\left|\lambda^{n} P_{i j}^{n} P_{j_{1 j}}^{n} C_{j}^{n}\right|+\left|\lambda^{n} P_{i j}^{n}\left(P_{i j}^{n}-1\right) C_{j}^{n}\right| \\
& +\sum_{\substack{i_{1}=1 \\
i_{1} \neq i}}^{M}\left|\lambda^{n} P_{i j}^{n} P_{i_{1} j}^{n} C_{j}^{n}\right|+\sum_{n_{1}=1}^{M} \sum_{j_{1}=1}^{J}\left|a_{j}^{n n_{1}} P_{i j}^{n}\right|+\left|P_{i j}^{n}\right| .
\end{aligned}
$$

All the terms in the expression of ${ }^{2} S_{i j}^{n}$ are positive since $0<P_{i j}^{n}<1$ and $\lambda^{n}, C_{j}^{n}, a_{j}^{n n_{1}} \geq 0$. Further factorizing ${ }^{1} S_{j}^{n}$ and ${ }^{2} S_{i j}^{n}$ and letting $\sum_{n_{1}=1}^{M} a_{j}^{n n_{1}} \leq$ $\mathbf{A}<\infty$, we have,

$$
\begin{aligned}
& { }^{1} S_{j}^{n}=2 \times \sum_{n_{1}=1}^{M} \sum_{j_{1}=1}^{J}\left|a_{j}^{n_{1} n} P_{j_{1} j}^{n_{1}}\left[1-\lambda^{n_{1}}\left\{b_{j_{1}}^{n_{1}}+d_{j_{1} j}^{n_{1}}-\sum_{l=1}^{J} P_{l j}^{n_{1}}\left(b_{l}^{n_{1}}+d_{l j}^{n_{1}}\right)\right\}\right]\right| \\
& { }^{2} S_{i j}^{n}=2^{3} \lambda^{n} P_{i j}^{n} C_{i j}^{n}\left(1-P_{i j}^{n}\right)+P_{i j}^{n}\left(J \sum_{n_{1}=1}^{M} a_{j}^{n n_{1}}+1\right) \\
& \leq 2^{3} \lambda^{n} P_{i j}^{n} C_{i j}^{n}\left(1-P_{i j}^{n}\right)+P_{i j}^{n}(J \mathbf{A}+1) .
\end{aligned}
$$

Let $\bar{p}_{j}^{n_{1}}=\sum_{l=1}^{J} P_{i j}^{n_{1}}\left(b_{l}^{n_{1}}+d_{l j}^{n_{1}}\right)$, then factorizing eqn. (12a) we have,

$$
\begin{aligned}
{ }^{1} S_{j}^{n} & \leq 2 \sum_{n_{1}=1}^{M} \sum_{j_{1}=1}^{J}\left|a_{j}^{n_{1} n}\right|\left|P_{j_{1} j}^{n_{1}}\left[1-\lambda^{n_{1}}\left\{b_{j_{1}}^{n_{1}}+d_{j_{1} j}^{n_{1}}-\bar{p}_{j}^{n_{1}}\right\}\right]\right| \\
& =2 \sum_{n_{1}=1}^{M}\left|a_{j}^{n_{1} n}\right|\left(\sum_{j_{1}=1}^{J}\left|P_{j_{1} j}^{n_{1}}\left[1-\lambda^{n_{1}}\left\{b_{j_{1}}^{n_{1}}+d_{j_{1} j}^{n_{1}}-\bar{p}_{j}^{n_{1}}\right\}\right]\right|\right) \\
& \leq 2 \sum_{n_{1}=1}^{M} \sum_{j_{1}=1}^{J}\left|P_{j_{1} j}^{n_{1}}\left[1-\lambda^{n_{1}}\left\{b_{j_{1}}^{n_{1}}+d_{j_{1} j}^{n_{1}}-\bar{p}_{j}^{n_{1}}\right\}\right]\right|
\end{aligned}
$$


The above result is obtained using Holder's inequality and properties of norm monotonicity, and further noting that $\sum_{n_{1}=1}^{M}\left|a_{j}^{n_{1} n}\right|<1, \forall n, j$ (refer to Section 2.1 for explanation).

To show that the sequence of states in eqn. (5) is a contraction we need to show that,

$$
\begin{aligned}
& \sqrt{4 M+6 J} \sup _{\delta}\|\nabla \mathbf{h}(\delta)\|_{\infty}<1 \\
& \Rightarrow \sqrt{4 M+6 J} {\left[\sup _{\delta}\left(\max _{\substack{n \in \mathbb{N}[1, M] \\
i, j \in \mathbb{N}[i, j]}}\left({ }^{1} S_{j}^{n},{ }^{2} S_{i j}^{n}\right)\right)\right]<1 } \\
& \Rightarrow \sqrt{4 M+6 J} {\left[\operatorname { s u p } _ { \delta } \left(\operatorname { m a x } _ { \substack { n \in \mathbb { N } [ 1 , M ] \\
i , j \in \mathbb { N } [ i , j ] } } \left(2 \sum_{n_{1}=1}^{M} \sum_{j_{1}=1}^{J}\left|P_{j_{1} j}^{n_{1}}\left[1-\lambda^{n_{1}}\left\{b_{j_{1}}^{n_{1}}+d_{j_{1 j}}^{n_{1}}-\bar{p}_{j}^{n_{1}}\right\}\right]\right|,\right.\right.\right.} \\
&\left.\left.\left.2^{3} \lambda^{n} P_{i j}^{n} C_{i j}^{n}\left(1-P_{i j}^{n}\right)+P_{i j}^{n}(J \mathbf{A}+1)\right)\right)\right]<1 .
\end{aligned}
$$

Point to note is that the arguments of the max function in the above equation are uniformly continuous $\forall \lambda^{n}$. As $\lambda^{n} \rightarrow \infty, P_{i j}^{n} \rightarrow 0$, hence it is easy to see that,

$$
\lim _{\lambda^{1}, \lambda^{2}, \ldots, \lambda^{n} \rightarrow \infty} 2 \sum_{n_{1}=1}^{M} \sum_{j_{1}=1}^{J}\left|P_{j_{1} j}^{n_{1}}\left[1-\lambda^{n_{1}}\left\{b_{j_{1}}^{n_{1}}+d_{j_{1} j}^{n_{1}}-\bar{p}_{j}^{n_{1}}\right\}\right]\right|=0
$$

and

$$
\lim _{\lambda^{1}, \lambda^{2}, \ldots, \lambda^{n} \rightarrow \infty} 2^{3} \lambda^{n} P_{i j}^{n} C_{i j}^{n}\left(1-P_{i j}^{n}\right)+P_{i j}^{n}(J \mathbf{A}+1)=0 .
$$

Therefore, we can choose large enough $\lambda^{n}, n=1, \ldots, M$, such that both the arguments of the max function in eqn. (14) can be made arbitrarily small $\forall \delta \in(\mathbf{0}, \mathbf{v})$. Hence, we can say that the sets,

$$
\Lambda_{S_{1}}:=\left\{\lambda^{n_{1}}: \lambda^{n_{1}} \in \Re, 2 \sum_{n_{1}=1}^{M} \sum_{j_{1}=1}^{J}\left|P_{j_{1} j}^{n_{1}}\left[1-\lambda^{n_{1}}\left\{b_{j_{1}}^{n_{1}}+d_{j_{1 j}}^{n_{1}}-\bar{p}_{j}^{n_{1}}\right\}\right]\right|<1\right\}
$$

and

$$
\Lambda_{S_{2}}:=\left\{\lambda^{n}: \lambda^{n} \in \mathfrak{R}, 2^{3} \lambda^{n} P_{i j}^{n} C_{i j}^{n}\left(1-P_{i j}^{n}\right)+P_{i j}^{n}(J \mathbf{A}+1)<1\right\},
$$


are nonempty. So clearly,

$$
\Lambda:=\left\{\lambda^{n}: \lambda^{n} \in \Re, \sqrt{4 M+6 J}\left[\sup _{\delta \in(\mathbf{0}, \mathbf{v})}\left(\max _{\substack{n \in \mathbb{N}[1, M] \\ i, j \in \mathbb{N}[i, j]}}\left({ }^{1} S_{j}^{n},{ }^{2} S_{i j}^{n}\right)\right)\right]<1\right\} \neq \emptyset
$$

Hence, letting

$$
\sqrt{4 M+6 J}\left[\sup _{\delta \in(\mathbf{0}, \mathbf{v})}\left(\max _{\substack{n \in \mathbb{N}[1, M] \\ i, j \in \mathbb{N}[i, j]}}\left({ }^{1} S_{j}^{n},{ }^{2} S_{i j}^{n}\right)\right)\right]<\mathbf{L} \leq 1
$$

Hence we can write eqn. (10) as

$$
\left|\left[\begin{array}{l}
\mathbf{g}_{1}(\mathbf{b}, \mathbf{x} ; \mathbf{d}, \mathbf{Y}) \\
\mathbf{g}_{2}(\mathbf{b}, \mathbf{x} ; \mathbf{d}, \mathbf{Y})
\end{array}\right]\right|_{2}<\mathbf{L} \cdot\left|\left[\begin{array}{llll}
\mathbf{b} & \mathbf{x} & \mathbf{d} & \mathbf{Y}
\end{array}\right]^{\top}\right|_{2} .
$$

Lemma 4. There exists a class $\mathcal{K}_{\infty}$ function $\alpha_{3}$, and a class $K$ function $\beta$ such that $V(\cdot, \cdot)$ in eqn. (4) satisfies Property 2 of an ISS-Lyapunov function.

Proof: From eqn. (16) we have,

$$
\begin{aligned}
\sum_{n=1}^{M} \sum_{j=1}^{J}\left(g_{1 j}^{n}\right)^{2}+\sum_{n=1}^{M} \sum_{i=1}^{J} \sum_{j=1}^{J}\left(g_{2 i j}^{n}\right)^{2} & <\mathbf{L}\left(\sum_{j=1}^{J} \sum_{n=1}^{M}\left(b_{j}^{n}\right)^{2}+\sum_{i=1}^{J} \sum_{j=1}^{J} \sum_{n=1}^{M}\left(x_{i j}^{n}\right)^{2}\right. \\
& \left.+\sum_{i=1}^{J} \sum_{j=1}^{J} \sum_{n=1}^{M}\left(d_{i j}^{n}\right)^{2}+\sum_{j=1}^{J} \sum_{n=1}^{M}\left(Y_{j}^{n}\right)^{2}\right)
\end{aligned}
$$

The left hand side of Property 2 for the RUBMRIO model given by eqn. (5), with the candidate ISS-Lyapunov function in eqn. (4), can be written as,

$$
\begin{aligned}
& V\left(\left|g_{1 j}^{n}\right|,\left|g_{2 i j}^{n}\right|\right)-V\left(\left|b_{j}^{n}\right|,\left|x_{i j}^{n}\right|\right)=\sum_{j=1}^{J} \sum_{n=1}^{M}\left[\left(g_{1 j}^{n}\right)^{2}-\left(b_{j}^{n}\right)^{2}\right]+\sum_{i=1}^{J} \sum_{j=1}^{J} \sum_{n=1}^{M}\left[\left(g_{2 i j}^{n}\right)^{2}-\left(x_{i j}^{n}\right)^{2}\right] \\
< & \underbrace{-[1-\mathbf{L}]\left(\sum_{j=1}^{J} \sum_{n=1}^{M}\left(b_{j}^{n}\right)^{2}+\sum_{i=1}^{J} \sum_{j=1}^{J} \sum_{n=1}^{M}\left(x_{i j}^{n}\right)^{2}\right)}_{\alpha_{3} \text { (class } \mathcal{K}_{\infty} \text { function) }}+\underbrace{\mathbf{L}\left(\sum_{i=1}^{J} \sum_{j=1}^{J} \sum_{n=1}^{M}\left(d_{i j}^{n}\right)^{2}+\sum_{j=1}^{J} \sum_{n=1}^{M}\left(Y_{j}^{n}\right)^{2}\right)}_{\beta \text { (class } \mathcal{K} \text { function) }} \\
= & -\alpha_{3}\left(\left|b_{j}^{n}\right|,\left|x_{i j}^{n}\right|\right)+\beta\left(\left|d_{i j}^{n}\right|,\left|Y_{j}^{n}\right|\right),
\end{aligned}
$$

where $\alpha_{3}\left(\left|b_{j}^{n}\right|,\left|x_{i j}^{n}\right|\right)$ and $\beta\left(\left|d_{i j}^{n}\right|,\left|Y_{j}^{n}\right|\right)$ are class $\mathcal{K}_{\infty}$ and class $\mathcal{K}$ functions as defined above. This shows that the candidate ISS-Lyapunov function in eqn. (4) satisfies Property 2 of an ISS-Lyapunov function. 
Theorem 2. The function $V(\cdot, \cdot)$ in eqn. (4) is an ISS-Lyapunov function and hence the fixed point of the RUBMRIO model given by eqn. (2) is stable.

Proof: From Lemma 2 and Lemma 4, we can see that $V(\cdot, \cdot)$ in eqn. (4) is an ISS-Lyapunov function for the system in eqn. (2). Now using Lemma 1, it can be said that the fixed point of the discrete dynamical system in eqn. (2) is ISS.

\subsection{Discussion}

The above analysis in Section 3.2 implies, for the solution of the RUBMRIO model to be ISS the dispersion parameters should be in the set defined in eqn. (15). The economic interpretation of this is, to guarantee input-to-state stability of eqn. (2) the commodity flows should be local and concentrated. The majority of the flow to the destination region should originate in the region offering the minimum total cost, defined as $p_{i j}^{n}=b_{j}^{n}+d_{i j}^{n}$ (see (Zhao and Kockelman, 2004), Section 3.1.2 for explanation).

For arbitrary values of the dispersion parameter $\lambda^{n}$, the purchase probability, defined as $P_{i j}^{n}$ here, is small only when the total cost $p_{i j}^{n}$ is sufficiently large, such that,

$$
\lim _{p_{i j} \rightarrow \infty} 2 \sum_{n_{1}=1}^{M} \sum_{j_{1}=1}^{J}\left|P_{j_{1} j}^{n_{1}}\left[1-\lambda^{n_{1}}\left\{b_{j_{1}}^{n_{1}}+d_{j_{1} j}^{n_{1}}-\bar{p}_{j}^{n_{1}}\right\}\right]\right|=0,
$$

and

$$
\lim _{p_{i j}^{n} \rightarrow \infty} 2^{3} \lambda^{n} P_{i j}^{n} C_{i j}^{n}\left(1-P_{i j}^{n}\right)+P_{i j}^{n}(J \mathbf{A}+1)=0 .
$$

for all values of $n=1, \ldots, M$ and $i, j=1, \ldots, J$. If the above happens, it is easy to see that the values of ${ }^{1} S_{j}^{n}$ and ${ }^{2} S_{i j}^{n}$ are sufficiently small such that,

$$
\sqrt{4 M+6 J}\left[\sup _{\delta \in(\mathbf{0}, \mathbf{v})}\left(\max _{\substack{n \in \mathbb{N}[1, M] \\ i, j \in \mathbb{N}[i, j]}}\left({ }^{1} S_{j}^{n},{ }^{2} S_{i j}^{n}\right)\right)\right]<1
$$

Hence we can say that for sufficiently large value of total $\operatorname{cost} p_{i j}^{n}$ the solution of the discrete dynamical system in eqn. (2) is ISS.

However, nothing can be concluded regarding input-to-state stability of the solutions of eqn. (2), if both the dispersion parameter $\lambda^{n}$ and the total cost $p_{i j}^{n}$ are arbitrary. 


\section{Inverse Identification Methodology for RUBMRIO Model Based on Optimal Control}

The method of inverse identification of the RUBMRIO model inputs derives its roots from the theory of inverse problems (Tarantola, 2002). In this work, inverse identification done by posing the problem of "calibrating the exogenous inputs" of the RUBMRIO model as an optimization problem. The resulting optimization problem is then solved in optimal control framework. For the sake of brevity the authors have decided to omit discussion on inverse problems. Interested readers may consult (Hansen, 2010) for introductory materials regarding inverse problems. However, in the sections following, we have discussed the motivation behind proposing such a methodology in the context in RUBMRIO models and the significant advantages it provides.

\subsection{Motivation}

The original iterative solution to the RUBMRIO model finds the commodity flows and the production costs, $x_{i j}^{n}$ and $b_{j}^{n}$ respectively in eqn. (2), given the exogenous inputs ( which are the final demand and the transportation costs here) specified by the social planner. In the context of inverse identification we will call it as forward model. The solution obtained by solving the forward model is called as the forward solution. However often a desired level of steady state commodity flow or production cost is sought. Here the social planner's task is to calibrate the RUBMRIO model such that the exogenous inputs that he/ she decides, leads to the desired level of commodity flows and production costs. We will call it as the inverse problem. The exogenous input levels found by solving the inverse problem is called as inverse solution.

To this end, we were interested in only the forward model, where the social planner's job was to provide the exogenous input values. The forward solutions were obtained when the RUBMRIO model reached a steady state solution. Inverse solutions are found by solving an optimization problem where the objective is that the optimal flows and production costs meet the desired level of flows and production costs. The optimal solution is constrained such that it satisfies the original RUBMRIO iterative equations. Thus the proposed inverse identification methodology finds the exogenous inputs that will yield the desired level of commodity flows and production costs when the forward model is solved, up to an acceptable order of accuracy. 
However, the proposed inverse identification methodology will yield meaningful solutions, only if solution exists for the optimal control problem. In the present work, we will operate under this caveat, whenever we formulate the optimal control problem for inverse identification of inputs. Interested readers are directed towards Ref. (Bryson and Ho, 1975) for an in-depth analysis on existence and uniqueness of solutions to optimal control problems.

\subsection{Advantages of Using an Inverse Identification Methodology}

Traditionally, for RUBMRIO model, the social planner chooses the exogenous inputs carefully and checks by solving the forward model, if the outputs of the RUBMRIO model match the one that the social planner expects. This is often a delicate task requiring years of expertise and is also time consuming (Dutta et al., 2014). The goal of inverse identification methodology is to help the social planner choose inputs. Formulating the problem of designing the exogenous inputs as an optimal control problem, eases the task of calibration. Moreover, It is also a robust method as the inputs are solutions of an optimization problem. Once the formulation is complete, one can use readily available optimization solvers to solve the problem (Nocedal and Wright, 1999).

\subsection{Similarity to Supply-Driven Models}

The technique of inverse identification presented here has some similarities to supply-driven models, like Ghosh's model (Ghosh, 1958), in essence that it is an inverse identification of a RUBMRIO model which is demanddriven. But it is well known that Ghosh's model becomes implausible for market economies (Oosterhaven, 1988). The extension to Ghosh's model has been proved to be implausible for centrally planned economies too (Oosterhaven, 2012).

Hence, in this work we use equations of the RUBMRIO model which come from the original demand-driven input-output model proposed by Leontief (Leontief, 1953). The solution of proposed inverse identification problem is subject to it satisfying the RUBMRIO model.

\subsection{Problem Formulation}

For the current case, we find the control inputs, i.e. the final demand and the transportation costs given desired values for production costs and commodity flows. We have formulated two optimal control problems depicting two different scenarios, to find the inputs to the RUBMRIO model. However 
the inverse problem formalism for the RUBMRIO model is not limited to these two cases and can be extended to any number of scenarios.

\subsubsection{Scenario 1}

Here an optimal control problem is solved to design the control inputs and the parameters such that the steady state production costs $\hat{b}_{j}^{n}$ and the commodity flows $\hat{x}_{i j}^{n}$ obtained by solving eqn. (2) are close to the desired costs and flows given by $\bar{b}_{j}^{n}$ and $\bar{x}_{i j}^{n}$. Since we have assumed that the states of the RUBMRIO model in eqn. (2) are in $\mathcal{L}_{2}$, the formulation of the optimal control problem is posed as a $\mathcal{L}_{2}$ norm minimization problem, which is given by,

$$
\begin{aligned}
& \min _{\substack{Y_{k}^{m}, d_{r k}^{m} \\
m \in \mathbb{N}[1, M] \\
r, k \in \mathbb{N}[1, J]}} \sum_{n=1}^{M} \sum_{j=1}^{J}\left(\bar{b}_{j}^{n}-\hat{b}_{j}^{n}\right)^{2}+\sum_{n=1}^{M} \sum_{i=1}^{J} \sum_{j=1}^{J}\left(\bar{x}_{i j}^{n}-\hat{x}_{i j}^{n}\right)^{2}, \\
& \text { subject to, } \\
& \hat{b}_{j}^{n}=\sum_{m=1}^{M} a_{j}^{m n}\left[\sum_{i=1}^{J} \frac{\exp \left(-\lambda^{m}\left(\hat{b}_{i}^{m}+d_{i j}^{m}\right)\right)\left(\hat{b}_{i}^{m}+d_{i j}^{m}\right)}{\sum_{r=1}^{J} \exp \left(-\lambda^{m}\left(\hat{b}_{r}^{m}+d_{r j}^{m}\right)\right)}\right] \\
& \hat{x}_{i j}^{n}=\left[\sum_{m=1}^{M} a_{j}^{n m}\left(\sum_{l=1}^{J} \hat{x}_{j l}^{m}\right)+Y_{j}^{n}\right]\left[\begin{array}{l}
\exp \left(-\lambda^{n}\left(\hat{b}_{i}^{n}+d_{i j}^{n}\right)\right) \\
\sum_{r=1}^{J} \exp \left(-\lambda^{n}\left(\hat{b}_{r}^{n}+d_{r j}^{n}\right)\right)
\end{array}\right]
\end{aligned}
$$

As stated in Section 2.1, we find the final demands $Y_{k}^{m}$ and the transportation costs $d_{r k}^{m}, m \in \mathbb{N}[1, M]$ and $r, k \in \mathbb{N}[1, J]$, such that the desired objective in eqn. (19a) is met, while satisfying the constraints in eqn. (19b).

\subsubsection{Scenario 2}

In this scenario, the control inputs (final demand and transportation costs) are found such that the commodity flow and the production prices track pre specified trajectories. Let the trajectories of the state variables are given by $\bar{b}_{j}^{n}(k)$ and $\bar{x}_{i j}^{n}(k)$ where $k=1, \ldots, N$ is the iteration counter with $N$ 
being the maximum number of iteration allowed. The optimization problem is then formulated as

$$
\min _{\substack{Y_{k}^{m}, d_{r s}^{m} \\ m \in \mathbb{N}[1, M] \\ r, s \in \mathbb{N}[1, J]}} \sum_{k=1}^{N}\left[\sum_{n=1}^{M} \sum_{j=1}^{J}\left(\bar{b}_{j}^{n}(k)-b_{j}^{n}(k)\right)^{2}+\sum_{n=1}^{M} \sum_{i=1}^{J} \sum_{j=1}^{J}\left(\bar{x}_{i j}^{n}(k)-x_{i j}^{n}(k)\right)^{2}\right],
$$

subject to,

$$
\begin{aligned}
& b_{j}^{n}(k+1)=\sum_{m=1}^{M} a_{j}^{m n}\left[\sum_{i=1}^{J} \frac{\exp \left(-\lambda^{m}\left(b_{i}^{m}(k)+d_{i j}^{m}\right)\right)\left(b_{i}^{m}(k)+d_{i j}^{m}\right)}{\sum_{r=1}^{J} \exp \left(-\lambda^{m}\left(b_{r}^{m}(k)+d_{r j}^{m}\right)\right)}\right] \\
& x_{i j}^{n}(k+1)=\left[\sum_{m=1}^{M} a_{j}^{n m}\left(\sum_{l=1}^{J} x_{j l}^{m}(k)\right)+Y_{j}^{n}\right]\left[\frac{\exp \left(-\lambda^{n}\left(b_{i}^{n}(k)+d_{i j}^{n}\right)\right)}{\sum_{r=1}^{J} \exp \left(-\lambda^{n}\left(b_{r}^{n}(k)+d_{r j}^{n}\right)\right)}\right]
\end{aligned}
$$

\subsection{Solution Methodology}

The problem given in eqn. (19) can be solved using general constrained optimization techniques. A nonlinear programming solver can be used to solve the problem. Details of how the solution can be obtained via nonlinear programming has been omitted here and can be found in Ref. (Nocedal and Wright, 1999).

The second problem in eqn. (20) can be solved using discrete time optimal control techniques. Let us represent the discrete dynamical system as in eqn. (5), which for the present case are the constraints. In the context of the current work, let $\mathbf{y}_{k}=[\mathbf{b}(k) \mathbf{x}(k)]$ be the states of the RUBMRIO model and $\mathbf{u}=[\mathbf{d}, \mathbf{Y}]$ be the control inputs. Then without loss of generality, the 
optimization problem in eqn. (20) can be then written as,

$$
\begin{aligned}
& \min _{\mathbf{u}} \sum_{k=1}^{N} \phi\left(\mathbf{y}_{k}\right) \\
& \text { subject to, } \\
& \mathbf{y}_{k+1}=\mathbf{h}\left(\mathbf{y}_{k}, \mathbf{u}\right)
\end{aligned}
$$

where $\mathbf{h}$ has the same meaning as in Lemma 3. Further, augmenting the objective function $\phi\left(\mathbf{y}_{k}\right)$ using KKT multipliers $\Gamma_{k}^{T}$, we have,

$$
\mathbf{L}=\phi\left(\mathbf{y}_{N}\right)+\sum_{k=1}^{N-1}\left[\phi\left(\mathbf{y}_{k}\right)+\Gamma_{k+1}^{\top}\left(\mathbf{y}_{k}-\mathbf{h}\left(\mathbf{y}_{k}, \mathbf{u}\right)\right)\right]
$$

The optimal $\mathbf{u}$ for the above problem is found using Pontryagin's minimum principle (Athans, 1967) which translates to solving the following system of equations simultaneously,

$$
\begin{aligned}
& \mathbf{y}_{k+1}=\mathbf{h}\left(\mathbf{y}_{k}, \mathbf{u}\right) \\
& \Gamma_{k}=\left(\frac{\partial \mathbf{h}\left(\mathbf{y}_{k}, \mathbf{u}\right)}{\partial \mathbf{y}_{k}}\right)^{\top} \Gamma_{k+1}+\frac{\partial \phi\left(\mathbf{y}_{k}\right)}{\partial \mathbf{y}_{k}} \\
& 0=\left(\frac{\partial \mathbf{h}\left(\mathbf{y}_{k}, \mathbf{u}\right)}{\partial \mathbf{u}}\right)^{\top} \Gamma_{k+1}+\frac{\partial \phi\left(\mathbf{y}_{k}\right)}{\partial \mathbf{u}} \\
& 0=\left(\Gamma_{N}-\frac{\partial \phi\left(\mathbf{y}_{N}\right)}{\partial \mathbf{y}_{N}}\right)^{\top} d \mathbf{y}_{N}
\end{aligned}
$$

Details of how eqn. (23a)- (23d) are obtained can be found in Chapter 2 in Ref. (Bryson and Ho, 1975).

\section{Application of the Proposed Optimal Control Methodology}

In this section we demonstrate the proposed optimal control methodology to find inputs of a RUBMRIO model when applied to an example problem. The example that is used is same as the one in Ref. (Zhao and Kockelman, 2004). However, in the present case we consider the inputs, i.e. the transportation costs $\left(d_{i j}^{n}\right)$ and the final demand $\left(Y_{j}^{n}\right)$, to be unknowns. The inputs are found such that the states i.e. the commodity flows $\left(x_{i j}^{n}\right)$ and the production prices $\left(b_{j}^{n}\right)$ satisfy desired optimality criterion. The two scenarios presented in Section 4 are investigated. 
The number of economic sectors $(M)$ and the number of geographical zones $(J)$ in the example problem are 2 and 2, respectively. The technical coefficients $\left(a_{j}^{m n}\right)$ and the dispersion parameter $\left(\lambda^{n}\right)$ are assumed to be constants. Their values are given in Table 1.

Table 1: Values of $a_{j}^{m n}$ and $\lambda^{n}$.

\begin{tabular}{|c|c|c|}
\hline Variable & Value & Zones \\
\hline$a_{j}^{11}$ & 0.2 & $j=1,2$ \\
$a_{j}^{12}$ & 0.8 & $j=1,2$ \\
$a_{j}^{21}$ & 0.7 & $j=1,2$ \\
$a_{j}^{22}$ & 0.1 & $j=1,2$ \\
$\lambda^{1}$ & 15 & \\
$\lambda^{2}$ & 0.2 & \\
\hline
\end{tabular}

\subsection{Application of Scenario 1}

We first apply the optimal control problem presented in Section 4.4.1 to the example problem. Here our task is to solve the find control inputs that lead to a desired steady state value. The desired steady state values for each state are given in Table 2. These values are the same as the steady state values obtained in Ref. (Zhao and Kockelman, 2004). We will check to see if the optimal control methodology is able to predict the control input values needed to obtain the state state.

Table 2: Desired steady state values for $x_{i j}^{n}$ and $b_{j}^{n}$.

\begin{tabular}{|c|c|c|}
\hline & $\bar{x}_{i j}^{n}$ (in units of commodity) & $b_{j}^{n}($ in $\$ \mathrm{~s})$ \\
\hline \multirow{2}{*}{ Sector 1 } & $\bar{x}_{11}^{1}=307.072, \bar{x}_{12}^{1}=0.0$ & $b_{1}^{1}=18.958$ \\
& $\bar{x}_{21}^{1}=0.040, \bar{x}_{22}^{1}=1730.404$ & $\bar{b}_{2}^{1}=11.554$ \\
\hline \multirow{2}{*}{ Sector 2 } & $\bar{x}_{11}^{2}=132.386, \bar{x}_{12}^{2}=49.736$ & $b_{1}^{2}=18.875$ \\
& $\bar{x}_{21}^{2}=120.777, \bar{x}_{22}^{2}=1359.615$ & $\bar{b}_{2}^{2}=11.335$ \\
\hline
\end{tabular}

The optimal control problem given in eqn. (19) is first converted into a nonlinear programming (NLP) problem. Then NLP solvers are used to solve 
the resulting problem. In this work we used trust region based methods to solve the NLP problem. The NLP solver user here was fmincon from MATLAB $^{\circledR}$ (Branch and Grace, 1996).

Table 3, Column 2 shows the actual input values reported in Ref. (Zhao and Kockelman, 2004) that are used to get the steady state values in Table 2. The percentage errors of the initial guess values supplied to the optimization problem and the final optimized input values, from the actual input values are also presented. The percentage error is calculated as follows,

$$
E_{i}=\frac{u_{i}-u_{a}}{u_{a}} \times 100, \quad E_{o}=\frac{u_{o}-u_{a}}{u_{a}} \times 100,
$$

where $E_{i}$ and $E_{o}$ are initial and optimized percentage error respectively. $u_{a}$ is the actual control input value and $u_{i}$ and $u_{o}$ are initial and optimized input values, respectively.

We can see that there is a large error between initial guesses of inputs supplied to the optimal control problem and the actual values of inputs. However the final optimized values obtained after solving the optimal control problem in eqn. (19) are close to the actual values, for all the inputs except for

$d_{12}^{2}$. For a sanity check, the steady state values for the RUBMRIO model are found using the input values obtained after optimization. It is observed that the even with different values of $d_{12}^{1}$ same steady state levels are obtained. Hence, the inverse problem (finding the inputs from states) in this case has non unique solutions. The actual value of $d_{12}^{1}$ is recovered with better initial guess.

Figure 1 shows how the objective function and the normed difference between the control input values obtained from the NLP algorithm and the actual input values, vary with each iteration of the NLP solver.

\subsection{Application of Scenario 2}

For the optimization problem given in eqn. (20), we use the same example as used in the Section 5.1. The maximum number of iterations allowed is fixed to $N=100$. The reference trajectories for the state variables are set to the ones followed, when the values of the inputs are same as given in Table 3, Column 2.

Figure 2 shows plot for error between state trajectories before optimization and the reference state trajectory. Figure 3 shows the plot for error between the reference values and the optimized values of commodity flows and the production costs (which are the states), respectively. The optimized 
Table 3: Comparison of values of the control inputs before and after optimization to the actual input values used in Ref. (Zhao and Kockelman, 2004). $Y_{j}^{n}$ has the unit of units of commodity demanded and $d_{i j}^{n}$ has the unit of $\$$ s.

\begin{tabular}{|c|c|c|c|c|c|}
\hline Input & $\begin{array}{c}\text { Actual } \\
\text { values }\left(u_{a}\right)\end{array}$ & $\begin{array}{c}\text { Values before } \\
\text { optimization }\left(u_{i}\right)\end{array}$ & $\begin{array}{c}\text { Percentage } \\
\text { error }\left(E_{i}\right)\end{array}$ & $\begin{array}{c}\text { Values after } \\
\text { optimization }\left(u_{o}\right)\end{array}$ & $\begin{array}{c}\text { Percentage } \\
\text { error }\left(E_{o}\right)\end{array}$ \\
\hline$Y_{1}^{1}$ & 100 & 302.871 & $202.871 \%$ & 100.00 & $-1.28 \times 10^{-5} \%$ \\
$Y_{2}^{1}$ & 200 & 602.401 & $207.281 \%$ & 200.001 & $7.78 \times 10^{-4} \%$ \\
$Y_{1}^{2}$ & 20 & 61.456 & $201.201 \%$ & 20.001 & $3.27 \times 10^{-4 \%}$ \\
$Y_{2}^{2}$ & 50 & 150.426 & $200.851 \%$ & 50.001 & $2.78 \times 10^{-3} \%$ \\
\hline$d_{11}^{1}$ & 2 & 7.265 & $263.264 \%$ & 1.999 & $-0.008 \%$ \\
$d_{12}^{1}$ & 10 & 32.377 & $223.766 \%$ & 10.000 & $0.0002 \%$ \\
$d_{21}^{1}$ & 10 & 32.878 & $228.785 \%$ & 10.000 & $0.0009 \%$ \\
$d_{22}^{1}$ & 1 & 5.547 & $454.739 \%$ & 1.000 & $0.0026 \%$ \\
$d_{11}^{2}$ & 2 & 8.747 & $337.360 \%$ & 2.000 & $0.0074 \%$ \\
$d_{12}^{2}$ & 10 & 31.967 & $219.672 \%$ & 15.000 & $50.00 \%$ \\
$d_{21}^{2}$ & 10 & 30.107 & $201.071 \%$ & 1.000 & $-0.0004 \%$ \\
$d_{22}^{2}$ & 1 & 5.802 & $480.198 \%$ & 0.999 & $-0.0075 \%$ \\
\hline
\end{tabular}
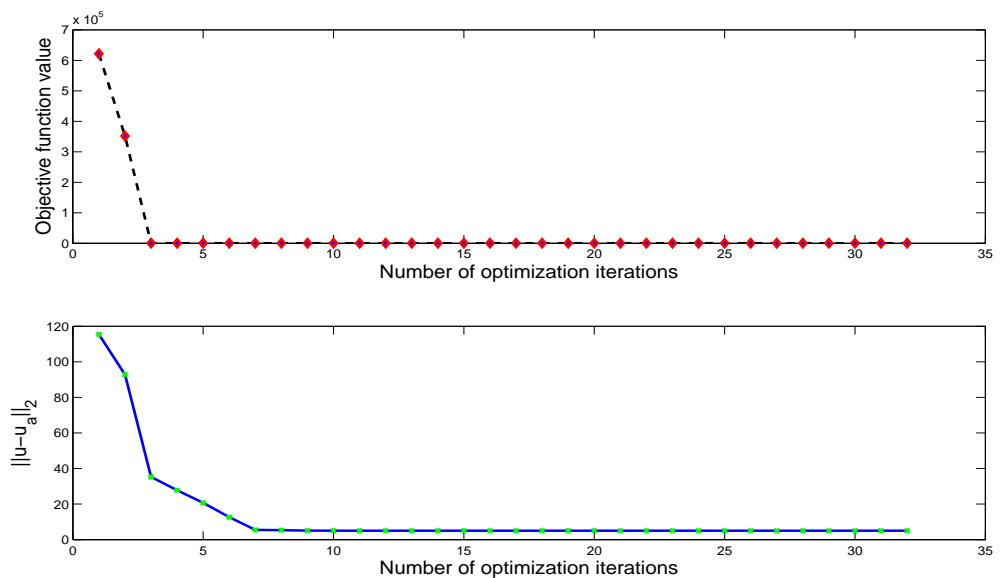

Figure 1: Variation of the objective function and the normed difference between calculated and the actual input values. $u$ refers to control input values obtained using the NLP solver at a given iteration. $u_{a}$ is the actual input value (Table 3 Column 2). 
values are the state values obtained after applying the proposed optimal control methodology described in Section 4.4.2.

It is observed that the proposed optimal control methodology is able to reduce error between reference and optimized state trajectory up to an acceptable level of accuracy. Hence it can be said that the optimal control methodology that is formulated in eqn. (20) is able to find inputs to the RUBMRIO model which will track a reference trajectory for the states.

\section{Conclusions and Scope for Future Work}

This work presents a novel methodology based on optimal control, to obtain inputs to the random utility based multiregional input-output (RUBMRIO) model given desired values for states. The states of the RUBMRIO model are the commodity flows and the production costs and the inputs are the final demand and the transportation costs.

At first it was shown that the RUBMRIO model can be formulated as a nonlinear discrete time dynamical system. Further it was proven that the resulting dynamical system is input-to-state stable. Then an optimal control methodology was postulated which would find the transportation costs and the final demand of the RUMBRIO model given desired commodity flow values and production costs.

The optimal control methodology was formulated for two different scenarios. Further they were applied to an example problem. For the first scenario, it was found that the proposed optimal control methodology could predict the all, but one of the inputs, upto an acceptable level of accuracy. For the second scenario, it was observed that the optimized control inputs could track a reference trajectory for the states accurately.

In future we are interested in applying the optimal control methodology to a large scale land use and transportation system, modeled based on random utility theory. The land use and transportation model would consists of several zones and sectors. Moreover, a comparison between traditional and the proposed inverse identification method for solving the RUBMRIO model will also be included. Further our goal is to generalize the optimal control methodology such that it can be applied to any land use and transportation model, regardless of the definition of the dynamical system. 

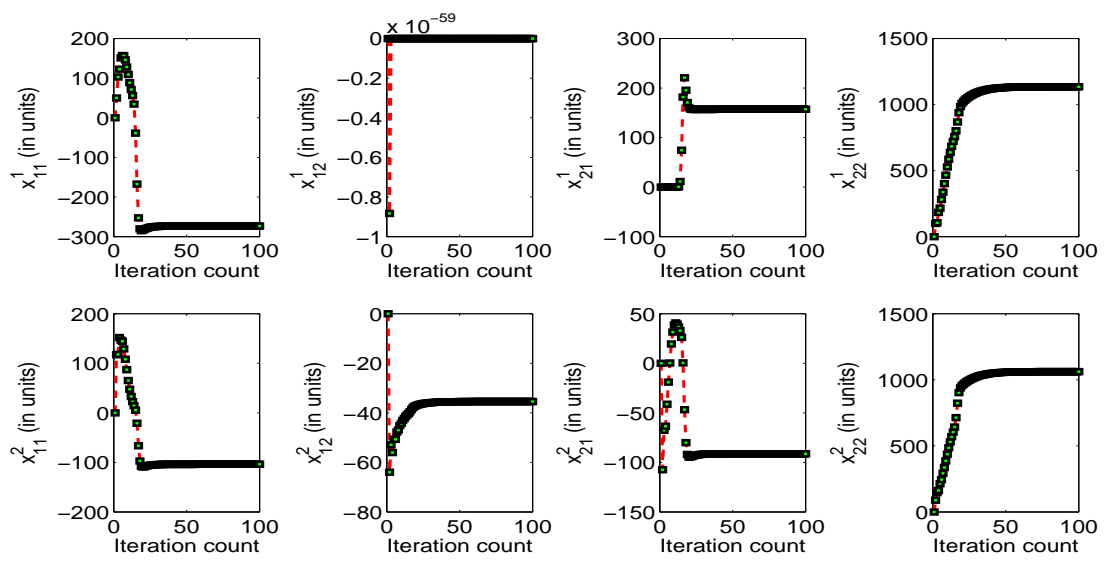

(a) Commodity flows.
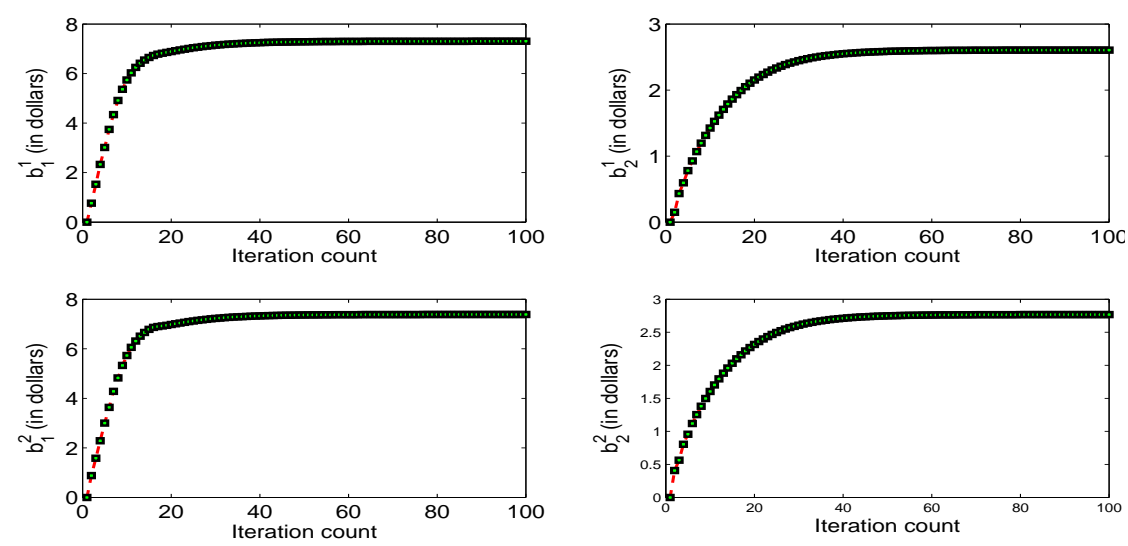

(b) Production costs.

Figure 2: Figure showing the error between reference and initial trajectories of the state variables. 

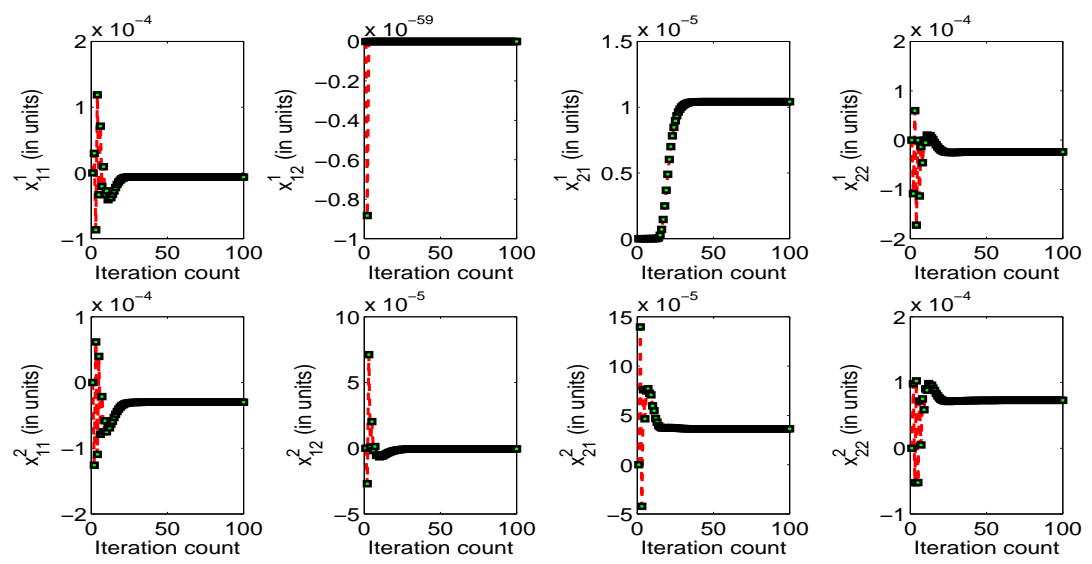

(a) Commodity flows.
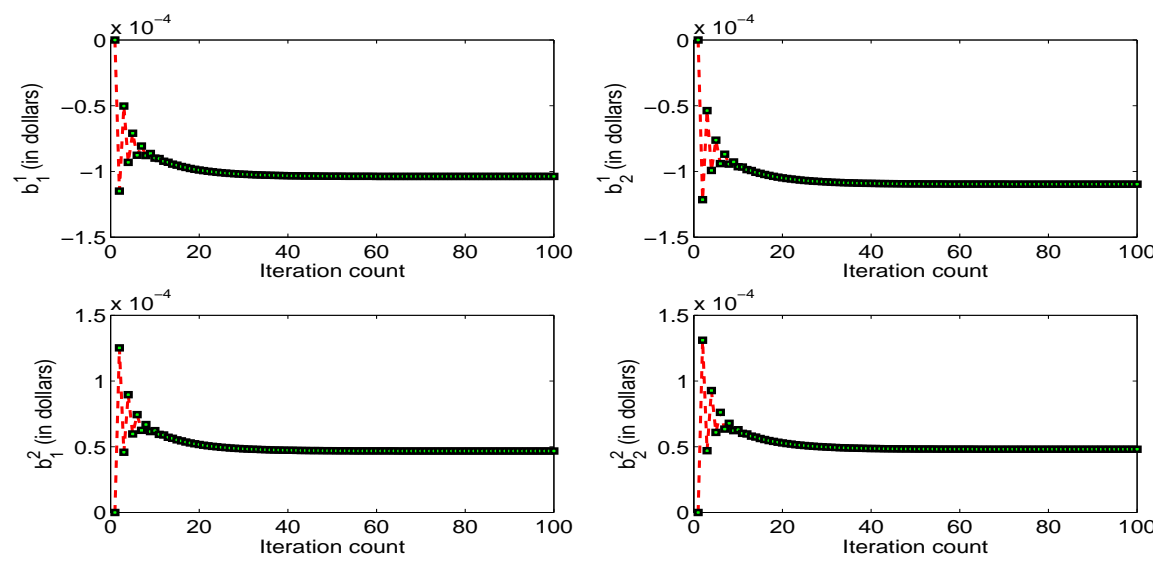

(b) Production costs.

Figure 3: Figure showing the error between reference and optimized trajetories of the state variables. 


\section{Acknowledgments}

The author of the manuscript would like to thank Dr. Kara Maria Kock-

elman for her immense help regarding the principles RUBMRIO model, and its working.

\section{References}

Athans, M., 1967. The matrix minimum principle. Information and control $11(5), 592-606$.

Ben-Naoum, L., Boel, R., Bongaerts, L., De Schutter, B., Peng, Y., Valckenaers, P., Vandewalle, J., Wertz, V., 1995. Methodologies for discrete event dynamic systems: a survey. Journal A: quarterly journal of automatic control 36 (4), 3-14.

Black, J. A., Paez, A., Suthanaya, P. A., 2002. Sustainable urban transportation: Performance indicators and some analytical approaches. Journal of Urban Planning and Development 128 (4), 184-209.

Branch, M. A., Grace, A., 1996. MATLAB: optimization toolbox: user's guide version 1.5. The MathWorks.

Bryson, A. E., Ho, Y.-C., 1975. Applied optimal control: Optimization, estimation, and control. Taylor \& Francis Group.

Chopard, B., Droz, M., 1998. Cellular automata modeling of physical systems. Vol. 122. Springer.

De La Barra, T., 1989. Integrated land use and transport modelling. Decision chains and hierarchies. No. 12. Cambridge University Press.

De La Barra, T., 2001. Integrated land use and transport modeling: The tranus experience. Planning support systems: Integrating geographic information systems, models and visualization tools. ESRI, Redlands CA, USA, $129-156$.

Dietzenbacher, E., 1997. In vindication of the ghosh model: a reinterpretation as a price model. Journal of regional science 37 (4), 629-651. 
Du, X., Kockelman, K. M., 2012. Tracking transportation and industrial production across a nation. Transportation Research Record: Journal of the Transportation Research Board 2269 (1), 99-109.

Dutta, P., Arnaud, E., Prados, E., Saujot, M., 2014. Calibration of an integrated land use and transportation model using maximum likelihood estimation. Computers, IEEE Transactions on 63 (1), 167-178.

Dutta, P., Arnaud, E., Prados, E., Saujot, M., Lefévre, B., 2012. Sensitivity analysis of a large scale integrated land use and transportation system. In: 3rd International Conference on Computational Sustainability.

Dutta, P., Bhattacharya, R., 2010. Nonlinear estimation of hypersonic state trajectories in bayesian framework with polynomial chaos. Journal of guidance, control, and dynamics 33 (6), 1765-1778.

Ghosh, A., 1958. Input-output approach in an allocation system. Economica, 58-64.

Hall, W., Newell, M., 1979. The mean value theorem for vector valued functions: A simple proof. Mathematics Magazine 52 (3), 157-158.

Hansen, P. C., 2010. Discrete inverse problems: insight and algorithms. Vol. 7. SIAM.

Huang, T., Kockelman, K. M., 2010. The introduction of dynamic features in a random-utility-based multiregional input-output model of trade, production, and location choice 47 (1).

Hunt, J. D., 1997. A Description of the MEPLAN Framework for Land Use and Transport Interaction Modelling. University of Calgary.

Jiang, Z., Wang, Y., 2001. Input-to-state stability for discrete-time nonlinear systems. Automatica 37 (6), 857-869.

Juri, N., Kockelman, K., 2004. Extending the random-utility-based multiregional input-output model: Incorporating land-use constraints, domestic demand and network congestion in a model of texas trade. In: At the Crossroads: Integrating Mobility Safety and Security. ITS America 2004, 14th Annual Meeting and Exposition. 
Kleinrock, L., 1975. Queueing systems. volume 1: Theory. Wiley-Interscience.

Leontief, W., 1986. Input-output economics. Oxford University Press on Demand.

Leontief, W. W., 1953. Studies in the Structure of the American Economy. Oxford University Press New York.

Nash, H. A., 2009. The european commission's sustainable consumption and production and sustainable industrial policy action plan. Journal of Cleaner Production 17 (4), 496-498.

Nocedal, J., Wright, S. J., 1999. Numerical optimization. Vol. 2. Springer New York.

Oosterhaven, J., 1988. On the plausibility of the supply-driven input-output model. Journal of Regional Science 28 (2), 203-217.

Oosterhaven, J., 2012. Adding supply-driven consumption makes the ghosh model even more implausible. Economic Systems Research 24 (1), 101-111.

Peirce, A. P., Dahleh, M. A., Rabitz, H., 1988. Optimal control of quantummechanical systems: Existence, numerical approximation, and applications. Physical Review A 37 (12), 4950.

Robinson, R. C., 2013. An introduction to dynamical systems: continuous and discrete. Vol. 19. American Mathematical Soc.

Ruiz Juri, N., Kockelman, K. M., 2006. Evaluation of the trans-texas corridor proposal: Application and enhancements of the random-utility-based multiregional input-output model 1. Journal of transportation engineering $132(7), 531-539$.

Sandefur, J. T., 1990. Discrete dynamical systems: Theory and applications. Oxford University Press, USA.

Sevcíková, H., Raftery, A. E., Waddell, P. A., 2007. Assessing uncertainty in urban simulations using bayesian melding. Transportation Research Part B: Methodological 41 (6), 652-669. 
Singh, B., Bhattacharya, R., Vadali, S. R., 2009. Verification of optimality and costate estimation using hilbert space projection. Journal of guidance, control, and dynamics 32 (4), 1345-1355.

Sontag, E., 2008. Input to state stability: Basic concepts and results. Nonlinear and optimal control theory, 163-220.

Sontag, E., Wang, Y., 1995. On characterizations of the input-to-state stability property. Systems \& Control Letters 24 (5), 351-359.

Spiekermann, K., Wegener, M., 2004. Evaluating urban sustainability using land-use transport interaction models. European Journal of Transport and Infrastructure Research 4 (3), 251-272.

Tarantola, A., 2002. Inverse problem theory: Methods for data fitting and model parameter estimation. Elsevier Science.

Zhao, Y., Kockelman, K., 2004. The random-utility-based multiregional input-output model: Solution existence and uniqueness. Transportation Research Part B: Methodological 38 (9), 789-807. 


\section{Appendix A. All Equations of the RUBMRIO Model}

$$
\begin{aligned}
\vartheta_{i j}^{n}(k+1) & =-\left(b_{i}^{n}(k)+d_{i j}^{n}+\epsilon_{i j}^{n}\right), \\
v_{i j}^{n}(k+1) & =-\left(b_{i}^{n}(k)+d_{i j}^{n}\right), \\
X_{i}^{m}(k+1) & =\sum_{j=1}^{J} x_{i j}^{m}(k), \\
C_{j}^{m}(k+1) & =\sum_{n=1}^{M} a_{j}^{m n} X_{j}^{n}(k+1)+Y_{j}^{m}, \\
x_{i j}^{n}(k+1) & =C_{j}^{n}(k+1) \frac{\exp \left(\lambda^{n} v_{i j}^{n}(k+1)\right)}{\sum_{r=1}^{J} \exp \left(\lambda^{n} v_{r j}^{n}(k+1)\right)} \\
c_{i j}^{n}(k+1) & =\frac{\sum_{i=1}^{J} x_{i j}^{n}(k+1)\left(b_{i}^{n}(k)+d_{i j}^{n}\right)}{J} \sum_{i=1}^{J} x_{i j}^{n}(k+1) \\
b_{j}^{n}(k+1) & =\sum_{m=1}^{M} a_{j}^{m n} \times c_{j}^{m}(k+1),
\end{aligned}
$$

- In eqn. (A.1a) $\vartheta_{i j}^{n}$ is the utility of purchasing a dollar worth of sector $n$ from region $i$ to use for production in region $j . \epsilon_{i j}^{n}$ is a random error term following i.i.d. Gumbel distribution.

- In eqn. (A.1b) $v_{i j}^{n}$ is called the systematic utility.

- In eqn. (A.1c) $X_{i}^{m}$ is the total commodity outflow of sectors $m$ in region $i$.

- In eqn. (A.1d) $C_{j}^{m}$ is the total consumption (inflow) of sector $m$ to region $j$.

- In eqn. (A.1f) $c_{j}^{n}$ is the average cost of using sector $n$ for production in region $j$. 